\title{
Profile of Patients With Tuberculous Pleural Effusion in Qatar: A Retrospective Study
}

Muhammad Zahid $^{1}$, Vamanjore A. Naushad ${ }^{2,3,4}$, Nishan K. Purayil ${ }^{5,}$ 3, 4 , Muhammad B. Jamshaid ${ }^{5}$, Jessiya Parambil ${ }^{5}$, Farah Rashid ${ }^{5}$, Shanima Ismail ${ }^{5}$, Muhammad Saddique ${ }^{5}$, Sajid Chalihadan ${ }^{5}$

1. Medicine, Hamad Medical Corporation, Doha, QAT 2. Internal Medicine, Hamad General Hospital/Hamad Medical Corporation, Doha, QAT 3. Internal Medicine, Weill Cornell Medical College, Doha, QAT 4. Internal Medicine, College of Medicine, Qatar University, Doha, QAT 5. Internal Medicine, Hamad Medical Corporation, Doha, QAT

Corresponding author: Vamanjore A. Naushad, nousha87@hotmail.com

\section{Abstract}

Introduction

Tuberculosis (TB) remains one of the top 10 causes of death globally. Around 1.7 billion people are infected with mycobacterium TB worldwide, and almost $90 \%$ of cases each year are found in 30 high TB burden countries. Due to the influx of a large expatriate population mainly from the high TB burden countries, there is an increased number of pulmonary ТВ as well as tuberculous pleural effusion cases reported in Qatar.

\section{Objectives}

The demographic, clinical, laboratory, and histopathological parameters of patients with tuberculous pleural effusion were assessed.

\section{Methods}

A single-center study was conducted at Hamad General Hospital, Hamad Medical Corporation, Doha, Qatar. Adults diagnosed to have tuberculous pleural effusion were included, and those with clinical suspicion of tuberculous pleural effusion with positive sputum acid-fast bacillus (AFB) but negative AFB in pleural samples were excluded.

\section{Results}

A total of 106 patients were reviewed, of whom 100 were included for the final analysis, with $86 \%$ being men. Majority were from the Asian subcontinent, and the mean age was 33.8 years ( $\mathrm{SD} \pm 10.3$ ). Main symptoms in decreasing order were cough (77\%), fever (56\%), and chest pain (54\%). Of the patients, $72 \%$ had normal BMI, and rest were above the normal range. Anemia and hypoalbuminemia were found in $36.7 \%$ and $89.8 \%$ of the patients, respectively. Positive AFB culture was observed in pleural biopsy (79\%), pleural fluid (13\%), and sputum (16\%). Positive AFB by polymerase chain reaction (PCR) was observed in pleural biopsy (57\%), pleural fluid (3\%), and sputum (2.2\%), whereas AFB smear was positive in $2 \%$ of pleural biopsy samples. Caseating granuloma was seen in $80 \%$ of patients. All the three Light's criteria were met by $30 \%$ of the

Review began 12/17/2020 Review ended 12/24/2020 Published 12/31/2020

\section{() Copyright 2020}

Zahid et al. This is an open access article distributed under the terms of the Creative Commons Attribution License CC-BY 4.0., which permits unrestricted use, distribution, and reproduction in any medium, provided the original author and source are credited. patients whereas $52 \%$ had two criteria fulfilled. No association between the number of Light's criteria and AFB yield was observed.

\section{Conclusions}

TPE was more common in healthy young adults. The AFB yield on pleural biopsy, PCR, and culture was significantly higher than that on all other samples. The number of positive Light's criteria did not have any association with positive AFB yield.

Categories: Internal Medicine, Infectious Disease

Keywords: tuberculosis, pleural effusion, bmi, lights criteria, tuberculous pleural effusion, acid-fast bacillus

\section{Introduction}

Tuberculosis (TB) remains one of the top 10 causes of death globally. According to the World Health Organization (WHO) 2019 report, around 1.7 billion people are infected with mycobacterium TB (MTB) globally, and almost $90 \%$ of cases each year are found in 30 high TB burden countries. The case rates vary from country to country, ranging from less than 50/million population/year to more than 5000/million population/year [1]. In 2018, there were 1.2 million deaths due to TB in human immunodeficiency virus (HIV) negative people. According to the WHO 2019 report, most cases are found in Southeast Asia (44\%), Africa (24\%), and the Western Pacific region (18\%). Eight countries accounted for two-thirds of total global cases of TB, that is, India (27\%), China (9\%), Indonesia (8\%), Philippines (6\%), Pakistan (6\%), Nigeria (4\%), Bangladesh (4\%), and South Africa (3\%) [2]. 
Extrapulmonary TB is not an uncommon entity, especially in areas where TB is endemic. Tuberculous pleural effusion (TPE) is one of the most common extrapulmonary TB after TB lymph node. One study carried out in the eastern part of Kingdom of Saudi Arabia reported that 37\% of pleural effusion were due to TB [3], whereas a study conducted in Qatar on 200 pleural effusion patients found that the most common cause of effusion was TB (32.5\%), and majority of them were from the Asian subcontinent [4]. The incidence of pleural involvement varies from $3-5 \%$ in TB non-endemic areas to $30 \%$ in TB endemic areas $[5,6]$.

The accumulation of fluid in TPE is multifactorial. The MTB antigen enters the pleural space following a rupture of subpleural caseous focus, which causes inflammatory response and results in increased capillary permeability. This also obstructs the lymphatic outflow, resulting in decreased clearance of pleural fluid [79]. TPE most commonly presents with fever (85\%), cough (70\%), and pleuritic chest pain (75\%). Dyspnea (50\%), night sweats (50\%), and weight loss (25\%) can also be there. TPE is commonly unilateral and the size of the effusion varies from patient to patient [10].

Even though Qatar is not listed in the high TB burden countries, a high number of pulmonary TB as well as TPE cases are reported due to the influx of a large expatriate population mainly from the high TB burden countries. In this single-center study, we sought to know the epidemiological, clinical, and biochemical profile of patients who were diagnosed with TPE.

\section{Materials And Methods}

\section{Study design and setting}

A retrospective observational study was carried out at the Hamad General Hospital, Hamad Medical Corporation, Doha, Qatar.

\section{Study population}

Patients older than 18 years of age who were admitted to the medical ward and diagnosed to have TPE between the period of January 2016 to December 2019 were included. The diagnosis of TPE was based on any of the following criteria: (1) positive acid-fast bacillus (AFB) by polymerase chain reaction (PCR)/smear or culture in the pleural fluid, (2) positive AFB by PCR/smear or culture in the pleural biopsy samples, and (3) caseating granuloma seen in pleural biopsy samples. Patients with clinical suspicion of TPE with positive sputum AFB but negative AFB in pleural samples were excluded.

\section{Study procedures}

Patient data were retrieved from the clinical information system using the health care number of study population. This included demographic features, body mass index (BMI), comorbid conditions, clinical symptom and signs, radiological data (chest X-ray, CT scan of the chest), blood investigations, pleural fluid analysis, and thoracoscopy /histopathology findings.

\section{Ethical consideration}

The study protocol was approved by the Ethics Committee of Medical Research Center of the institution.

\section{Statistical analysis}

Descriptive statistics were used to summarize and determine the sample characteristics and distribution of various considered parameters related to demographic and clinical features, biochemical parameters, and histopathological characteristics. The normally distributed data and results are reported with mean and standard deviation (SD). Categorical data are summarized using frequencies and percentages. Associations between two or more qualitative variables were examined and assessed using Pearson's chi-square test and Fisher's exact test as appropriate. Unpaired t-test and analysis of variance (ANOVA) were used to compare mean values of different quantitative parameters (such as age, BMI, white blood cell count, C-reactive protein (CRP), and other biochemical parameters) between two or more groups (such as AFB, histopathology, and treatment details). All statistical analyses were performed using statistical packages SPSS Version 24.0 (IBM Corp., Armonk, NY, USA) and Epi Info 2000 (Centers for Disease Control and Prevention, Atlanta, GA, USA).

\section{Results}

\section{Basic demography}

A total of 106 files were reviewed for inclusion, of which 100 patients fulfilled the criteria for analysis after exclusion (Incomplete data: 3; non-TB effusion: 3). Majority (86\%) of the patients were men, and the mean age was 33.85 years (range: 18 -84 years; $\mathrm{SD}^{ \pm} 10.26$ ). Of the study patients, $60 \%$ were from the Asian subcontinent. Cough (77\%), fever (56\%), and chest pain (54\%) were the most common presenting symptoms. Also, $72 \%$ had normal BMI (18.6-25). None of the patients was underweight (i.e., with BMI less than 18.5). The basic demographics and clinical features are summarized in Table 1 . 


\section{Cureus}

Characteristics

Gender

Male

Female

Age (mean $\pm S D$ ), years

Mean age

$<30$

$30-50$

$>50$

Length of stay (days $\pm S D$ )

Mean length of stay

Nationality

Qatar

Africa

India

Nepal

Sri Lanka

Bangladesh

Philippines

Indonesia

Pakistan

Childhood TB

Yes

No

Missing data

Contact with open TB

Yes

No

Missing data

Comorbidities

Nil

Diabetes

Hypertension

Asthma/COPD

Kidney Disease

HIV

Symptoms

Fever

Cough
Number (\%), n = 100

86 (86)

$14(14)$

$33.85 \pm 10.26$

$45(45)$

$50(50)$

$5(5)$

$8.34( \pm 3.54)$

1 (1)

$25(25)$

19 (19)

28 (28)

2 (2)

9 (9)

$13(13)$

1 (1)

2 (2)

3 (3.57)

84 (96.3)

13

$10(10.3)$

87 (89.7)

3

89

6 (6)

2 (2)

5 (5)

1 (1)

0

$56(56)$

77 (77) 


\section{Cureus}

\begin{tabular}{|c|c|}
\hline Weight loss & $25(25)$ \\
\hline Night sweats & $29(29)$ \\
\hline Chest pain & $54(54)$ \\
\hline SOB & $34(34)$ \\
\hline Anorexia & 15 (15) \\
\hline others & 17 (17) \\
\hline \multicolumn{2}{|l|}{ Duration of symptoms (days) } \\
\hline$<7$ & $26(26)$ \\
\hline 8 to 15 & $34(34)$ \\
\hline $16-30$ & $28(28)$ \\
\hline$>30$ & $12(12)$ \\
\hline \multicolumn{2}{|l|}{ BMI } \\
\hline$<18.5$ & 0 \\
\hline $18.6-25$ & $72(72)$ \\
\hline $25.1-30$ & $26(26)$ \\
\hline$>30$ & $2(2)$ \\
\hline \multicolumn{2}{|l|}{ PPD } \\
\hline$<5 \mathrm{~mm}$ & $14(34.1)$ \\
\hline $6-15 \mathrm{~mm}$ & $9(22)$ \\
\hline$>15 \mathrm{~mm}$ & $18(43.9)$ \\
\hline missing data & 59 \\
\hline \multicolumn{2}{|l|}{ QuantiFERON® } \\
\hline Positive & 37 (75.5) \\
\hline Negative & $12(24.5)$ \\
\hline Missing data & 51 \\
\hline \multicolumn{2}{|l|}{ Other TB manifestation } \\
\hline Parenchymal involvement & $10(10)$ \\
\hline Lymph nodes & $3(3)$ \\
\hline Pott's spine & $1(1)$ \\
\hline Pericardial & $1(1)$ \\
\hline Peritoneal & $3(3)$ \\
\hline Disseminated & $1(1)$ \\
\hline \multicolumn{2}{|l|}{ Drug resistance } \\
\hline No & 95 \\
\hline INH & 3 \\
\hline Rifampicin & 2 \\
\hline \multicolumn{2}{|l|}{ MDR-TB } \\
\hline No & 98 \\
\hline Yes & 2 \\
\hline
\end{tabular}




\section{Cureus}

\section{TABLE 1: Basic demographic characteristics and clinical profile}

TB, tuberculosis; COPD, chronic obstructive pulmonary disease; HIV, human immunodeficiency virus; SOB, shortness of breath; PPD, purified protein derivative; INH, isoniazid; MDR-TB, multidrug-resistant tuberculosis

\section{Laboratory parameters}

Around $36.7 \%$ of the patients had anemia. In $44.9 \%$, the platelet count was found to be high. Majority of the patients had hypoalbuminemia (89.8\%). CRP was elevated in all patients except three, and $53.9 \%$ of patients had a CRP of more than 100 (Table 2).

\begin{tabular}{|c|c|}
\hline Variable & Number (\%), $\mathrm{n}=100$ \\
\hline \multicolumn{2}{|l|}{ Hemoglobin } \\
\hline Normal & $62(63.3)$ \\
\hline Anemia & $36(36.7)$ \\
\hline Missing & 2 \\
\hline \multicolumn{2}{|c|}{ White blood cell count ( $\left.10^{3} / \mathrm{uL}\right)$} \\
\hline $4,000-10,000$ & $89(89.9)$ \\
\hline$<4,000$ & 0 \\
\hline$>10,000$ & $10(10.1)$ \\
\hline Missing data & 1 \\
\hline \multicolumn{2}{|l|}{ Lymphocytes } \\
\hline Normal & $59(60.2)$ \\
\hline Lymphopenia & 39 (39.8) \\
\hline Lymphocytosis & 0 \\
\hline Missing data & 2 \\
\hline \multicolumn{2}{|l|}{ Platelets (103/uL) } \\
\hline $150-400$ & $52(53.1)$ \\
\hline$>400$ & $44(44.9)$ \\
\hline$<150$ & $2(2)$ \\
\hline Missing data & 2 \\
\hline \multicolumn{2}{|c|}{ C-reactive protein (mg/L) } \\
\hline 0-5 & $3(3.4)$ \\
\hline $6-100$ & 38 (42.7) \\
\hline $101-200$ & 47 (52.8) \\
\hline >200 & $1(1.1)$ \\
\hline Missing data & 11 \\
\hline \multicolumn{2}{|l|}{ Albumin (gm/L) } \\
\hline $35-52$ & 10 (10.2) \\
\hline$<35$ & $88(89.8)$ \\
\hline Missing data & 2 \\
\hline \multicolumn{2}{|l|}{ CXR effusion } \\
\hline Right sided & $52(52)$ \\
\hline
\end{tabular}




\section{Cureus}

Left sided

$46(46)$

Bilateral

CXR: parenchymal involvement

Yes

Pleural fluid: lymphocytes

$>50 \%$

87 (88.8)

$<50 \%$

11 (11.2)

Missing data

2

Light's criteria

Protein ratio $>0.5$

97 (97)

$\mathrm{LDH}$ ratio $>0.6$

$52(52)$

LDH > two-thirds of serum LDH

$63(63)$

Number of Light's criteria fulfilled

1

18 (18)

2

$52(52)$

30 (30)

Therapeutic thoracocentesis

$19(19)$

Pleural biopsy: histopathology

Caseating/necrotizing granuloma

$80(80)$

Noncaseating granuloma

$13(13)$

Others

7 (7)

Thoracoscopic findings

Granuloma

11 (11.57)

Adhesions

$42(44.21)$

Inflamed pleura

77 (81.05)

Sago-like nodules/nodules

52 (4.74)

Incomplete data

Complication of thoracoscopy

Nil

89 (89)

Pneumothorax

9 (9)

Hydropneumothorax

2 (2)

\section{TABLE 2: Laboratory parameters and thoracoscopy findings}

CXR, chest X-ray; LDH, lactate dehydrogenase

\section{Radiological findings}

Of the study patients, $52 \%$ had right-sided pleural effusion and $46 \%$ had left-sided effusion on their chest Xrays. Only two patients had bilateral pleural effusion on their chest X-ray. Parenchymal involvement in chest 


\section{Cureus}

$\mathrm{X}$-ray was noted in $10 \%$ of the patients (Table 2).

\section{Pleural fluid analysis}

Overall, $87 \%$ of the patients had lymphocyte predominance (>50\% lymphocytes) in the pleural fluid, and almost all except three had a pleural fluid/serum protein ratio of more than 0.5 . On analyzing patients fulfilling the Light's criteria, all three criteria were met by $30 \%$ of the patients, whereas $52 \%$ had two criteria fulfilled. The details of pleural fluid analysis are summarized in Table 2.

\section{Thoracoscopy and pleural biopsy findings}

All patients underwent thoracoscopy. Inflamed pleura (81\%) and nodules (54.7\%) were the most common findings observed in thoracoscopy. On histopathological examination of the biopsy sample, necrotizing/caseating granuloma was seen in $80 \%$ of the study patients. Eleven patients developed complications after thoracoscopy (pneumothorax in nine and hydropneumothorax in two) (Table 2).

\section{AFB yield by different specimens and different methods}

The yield of AFB by smear was low in all three samples (sputum: 0; pleural fluid: 0; and pleural biopsy: $2 \%$ ). Even though the positivity of AFB by PCR technique in sputum and pleural fluid was low ( 2 and 3 , respectively), it was significantly high in biopsy samples (57\%). Similarly, AFB culture in biopsy specimen showed significantly higher positivity (79\%) than sputum or pleural fluid culture (15\% and $13 \%$, respectively) (Table 3).

\begin{tabular}{|c|c|c|c|}
\hline \multirow{2}{*}{$\begin{array}{l}\text { Variable } \\
\text { Sputum }\end{array}$} & \multicolumn{3}{|l|}{ AFB yield } \\
\hline & Positive & Negative & Missing data \\
\hline Smear & 0 & 92 & 8 \\
\hline PCR & 2 & 87 & 11 \\
\hline Culture & 15 & 78 & 7 \\
\hline \multicolumn{4}{|c|}{ Pleural fluid } \\
\hline Smear & 0 & 100 & \\
\hline PCR & 3 & 97 & \\
\hline Culture & 13 & 87 & \\
\hline \multicolumn{4}{|c|}{ Pleural biopsy } \\
\hline Smear & 2 & 98 & \\
\hline PCR & 57 & 43 & \\
\hline Culture & 79 & 21 & \\
\hline
\end{tabular}

\section{TABLE 3: AFB yield from various sources}

PCR, polymerase chain reaction: AFB, acid-fact bacillus

\section{Sputum AFB positivity with normal chest X-ray}

In patients with no obvious parenchymal involvement on chest X-ray, sputum for AFB was positive by PCR and culture in $2.2 \%(2 / 90)$ and $11.1 \%(10 / 90)$, respectively.

\section{Comparison of parameters based on Light's criteria}

We divided the patients into three groups based on the number of fulfilled Light's criteria and compared the laboratory parameters, mainly the positive yield of AFB, using different methods. There was no significant difference in the positive yield of AFB by different techniques in samples from sputum, pleural fluid, and pleural biopsy, and the three Light's criteria groups (Table 4). 


\section{Cureus}

Blood: WBC count $\left(10^{3} / \mathrm{uL}\right)$

More than 10,000

43

28

Lymphocytes

Normal

12

30

17

0.627

Lymphopenia

5

22

12

CRP (mg/L)

$0-5$

3

$6-100$

11

13

$101-200$

3

$>200$

1

Quanti-FERON

Negative

Positive

6

5

19

4

0.757

Mantoux (PPD)

$<5 \mathrm{~mm}$

3

6-15 mm

2

$>15 \mathrm{~mm}$

5

12

37

0.004

47

1

Chest X-ray: parenchymal involvement

No

13

47

30

0.008

Yes

5

5

0

90

10

0.932

BMI

$18.5-25$

39

25.1-30

13

18

72

$>30$

2

11

0.178

Serum albumin (gm/L)

Normal

3

6

0.276

Less than 35

14

46

28

Pleural fluid: lymphocyte

$>50 \%$

$<50 \%$

17

43

9

27

87

0.099

Pleural fluid AFB: smear

Positive

Negative

18

0

0

52

30

100

Pleural fluid: AFB PCR

Positive

0

3

3

Negative

18

49

30

Pleural fluid: AFB culture

Positive

4

8

44

1

13

Negative

14

29

0.129

041 


\section{Cureus}

Pleural biopsy: AFB smear

Positive

Negative

Pleural biopsy: AFB PCR

Positive

Negative

Pleural biopsy: AFB culture

Positive

Negative

Drug resistance

Nil

INH

Rifampicin

15

3

2

16

12

6

15

0
0

52

25

27

37

15
0

30

20

10

27

3

30

0

0
2

98

0.01

57

0.172

43

0.115

21

3

0.003

\section{TABLE 4: Correlation of variables with Light's criteria}

WBC, white blood cell; CRP, C-reactive protein; PPD, purified protein derivative; AFB, acid-fast bacillus; PCR, polymerase chain reaction; INH, isoniazid

\section{Discussion}

In this study, we sought to know the profile of patients with TPE in a tertiary care hospital in Qatar. All the study patients except one belonged to the expat population mainly from high TB burden countries. More than half of our study cohort was from the Asian subcontinent, mainly Nepal, India, followed by the African continent. The number of native Qatari nationals and patients from the western world with TPE were negligible. From the clinical point of view, most patients were young, healthy patients with no significant prior comorbid conditions and majority presented with cough and fever.

We observed that majority of our study population had normal BMI and few were in the overweight category. Contrary to the regular belief, none of the patients was underweight. Several studies have been conducted in the past on the association between BMI and TB and have shown conflicting results. A study conducted on two groups of children, one with pulmonary TB and the other with extrapulmonary TB, showed that the proportion of overweight or obese boys was higher in the group with extrapulmonary TB (32.4\%) compared to the group with pulmonary TB (13.8\%) [11]. Another study conducted on patients older than 65 years reported that obese and overweight persons have a significantly lower risk of developing active TB when compared to normal-weight individuals [12]. Others studies have shown the association between low BMI with high risk of TB [13] and severity of disease [14,15].

Almost half of our study patients had elevated platelet count. Platelet count and mean platelet volume (MPV) have been studied before as inflammatory markers in TB. One study showed significantly high platelet count and MPV in patients with active TB, which decreased with anti-tuberculous treatment [16], whereas, another study carried out by Lee et al. showed significantly high MPV and platelet count in TB. Their results revealed a direct correlation between MPV and CRP in TB but no correlation between platelet count and CRP [17]. The pathophysiological mechanism by which platelets acts as inflammatory cells includes activation of complements, release of inflammatory mediators, and increased vascular permeability [18].

Our results showed that the yield of AFB smear and PCR on pleural fluid was very low and negligible $(0 \%$ and 3\%, respectively). The literature showed that microscopic smear with Ziehl-Neelsen (ZN) stain of pleural fluid has a yield of fewer than $10 \%$ [19], which may increase to $20 \%$ in HIV-positive people with effusion, TB empyema, and loculated effusion [20,21]. Nucleic acid amplification test (NAAT) by PCR, which detects MTB genetic material, has a sensitivity ranging from $28 \%$ to $81 \%$ for pleural fluid and $90 \%$ for pleural tissue and a specificity of $90 \%$ to $100 \%$ [22]. Similar to our observation, previous studies conducted by Antonio et al. also showed a similar result. In their study, only two out of 79 patients were positive for ZN stain of pleural fluid, and eight (13.7\%) out of 52 patients were positive for PCR [23]. Low bacterial load and the presence of PCR inhibitors in pleural fluid have been shown as possible reasons for this low yield in pleural fluid [24]. The positive yield from pleural fluid AFB culture in our study was only $13 \%$, which is in agreement with the 
results of Antonio et al. (16.6\%) [23]. The yield of mycobacterial culture from pleural fluid varies according to the culture media, ranging from less than $30 \%$ in Lowenstein-Jensen medium culture to $70 \%$ in BACTECMGIT medium culture $[19,25]$.

Similar to pleural fluid, the yield of AFB smear in pleural biopsy was also low in our study (2\%), whereas yield of PCR and culture of pleural biopsy samples were high, with culture (79\%) showing a significantly higher positivity rate than PCR (57\%). This is in contrast to the results of a past study that reported a diagnostic accuracy of $23.5 \%$ by smear or PCR or culture of biopsy sample [23]. Histopathological results in our study showed a very high percentage of the presence of granuloma in the study samples (80\%), which is in contrast to the previously reported results of $41.2 \%$ [23]. Pleural biopsy has a sensitivity of $69 \%-97 \%$, which is higher in HIV-positive patients [20,22]. The technique used for obtaining biopsy also has an effect on sensitivity. Medical thoracoscopy shown to have a sensitivity of upto 100\% for TB pleuritis and increases the yield for culture and PCR [26,27], whereas ultrasound-guided biopsy has a diagnostic yield of 90\% [28].

We also evaluated the sputum AFB positivity in patients with no obvious parenchymal involvement in chest X-ray. It was observed that sputum sample was bacteriologically positive (PCR or culture) in 15\% (15/100) of patients with TPE, out of which $10 \%$ had no parenchymal involvement on chest X-ray. This has clinical significance in deciding the treatment regimen of the patient and also has epidemiological implications. In routine clinical practice, patients with TPE without obvious parenchymal involvement on chest X-ray are considered noninfectious. However, positive AFB in sputum in such patients renders them infectious, which needs isolation as well as contact tracing. In addition, the treatment regimen of such patients varies from that of TPE with negative sputum AFB. In comparison to our observation, previous studies have reported a higher percentage of positivity in sputum. Chaudhuri et al. evaluated the role of sputum examination for AFB in TPE patients without parenchymal involvement and reported that in $22.2 \%$ of their patients the sputum AFB was positive by smear and/ or culture [29]. Conde et al. studied the yield of AFB smear and culture in induced sputum in TPE and found that bacteriological yield was $55 \%$ for sputum culture in patients with normal chest X-ray [30].

\section{Conclusions}

In the state of Qatar, TPE was more common in young healthy adults from high TB burden countries. None of the study patients was underweight, giving the assumption that low BMI is not a vulnerability factor for TPE. The AFB yield on pleural biopsy PCR and culture was significantly higher than that on all other samples. The number of positive Light's criteria did not have any association with positive AFB yield. Finally, we recommend that sputum for AFB tests should be conducted in all patients with TPE even with no obvious parenchymal lesion on chest X-ray.

\section{Additional Information \\ Disclosures}

Human subjects: Consent was obtained by all participants in this study. Ethics Committee of Medical Research Center of HMC issued approval MRC 01-20-004. Animal subjects: All authors have confirmed that this study did not involve animal subjects or tissue. Conflicts of interest: In compliance with the ICMJE uniform disclosure form, all authors declare the following: Payment/services info: All authors have declared that no financial support was received from any organization for the submitted work. Financial relationships: All authors have declared that they have no financial relationships at present or within the previous three years with any organizations that might have an interest in the submitted work. Other relationships: All authors have declared that there are no other relationships or activities that could appear to have influenced the submitted work.

\section{Acknowledgements}

The authors are thankful to Dr. Osama Hashim Mohammad, Senior Consultant Department of General Internal Medicine, HMC, and Dr. Premchandra , Academic Research Scientist, Medical Research Center, HMC, for their valuable suggestion and support in the data analysis and interpretation.

\section{References}

1. Global tuberculosis report 2019: chapter 1. (2019). Accessed: October 1, 2020: https://apps.who.int/iris/bitstream/handle/10665/329368/9789241565714-eng.pdf.

2. Global tuberculosis report 2019: chapter 3. (2019). Accessed: October 1, 2020: https://apps.who.int/iris/bitstream/handle/10665/329368/9789241565714-eng.pdf.

3. Al-Qorain A, Larbi EB, al-Muhanna F, Satti MB, Baloush A, Falha K: Pattern of pleural effusion in Eastern Province of Saudi Arabia: a prospective study. East Afr Med J. 1994, 71:246-249.

4. Khan FY, Alsamawi M, Yasin M, et al.: Etiology of pleural effusion among adults in the state of Qatar: a 1year hospital-based study. East Mediterr Health J. 2011, 17:611-618.

5. Baumann MH, Nolan R, Petrini M, Lee YC, Light RW, Schneider E: Pleural tuberculosis in the United States: incidence and drug resistance. Chest. 2007, 131:1125-1132. 10.1378/chest.06-2352

6. Diacon AH, Van de Wal BW, Wyser C, Smedema JP, Bezuidenhout J, Bolliger CT, Walzl G: Diagnostic tools in tuberculous pleurisy: a direct comparative study. Eur Respir J. 2003, 22:589-591. 
10.1183/09031936.03.00017103a

7. Berger HW, Mejia E: Tuberculous pleurisy. Chest. 1973, 63:88-92. 10.1378/chest.63.1.88

8. Stead WW, Eichenholz A, Stauss HK: Operative and pathologic findings in twenty-four patients with syndrome of idiopathic pleurisy with effusion, presumably tuberculous. Am Rev Tuberc. 1955, 71:473-502.

9. Light RW: Update on tuberculous pleural effusion. Respirology. 2010, 15:451-458. 10.1111/j.14401843.2010.01723.x

10. Andreu J, Cáceres J, Pallisa E, Martinez-Rodriguez M: Radiological manifestations of pulmonary tuberculosis. Eur J Radiol. 2004, 51:139-149. 10.1016/j.ejrad.2004.03.009

11. L. Gorbach: Body mass index in children with tuberculosis . Am J Resp Crit Care Med. 2020, 201:5156.

12. Leung CC, Lam TH, Chan WM, et al.: Lower risk of tuberculosis in obesity . Arch Intern Med. 2007, 167:12971304. 10.1001/archinte.167.12.1297

13. Tverdal A: Body mass index and incidence of tuberculosis . Eur J Respir Dis. 1986, 69:355-362.

14. Van Lettow M, Kumwenda JJ, Harries AD, et al.: Malnutrition and the severity of lung disease in adults with pulmonary tuberculosis in Malawi. Int J Tuberc Lung Dis. 2004, 8:211-217.

15. Zachariah R, Spielmann MP, Harries AD, Salaniponi FM: Moderate to severe malnutrition in patients with tuberculosis is a risk factor associated with early death. Trans R Soc Trop Med Hyg. 2002, 96:291-294. 10.1016/s0035-9203(02)90103-3

16. Tozkoparan E, Deniz O, Ucar E, Bilgic H, Ekiz K: Changes in platelet count and indices in pulmonary tuberculosis. Clin Chem Lab Med. 2007, 45:1009-1013. 10.1515/CCLM.2007.194

17. Lee MY, Kim YJ, Lee HJ, Cho SY, Park TS: Mean platelet volume in Mycobacterium tuberculosis Infection . Biomed Res Int. 2016, 2016:7508763. 10.1155/2016/7508763

18. Jenne CN, Urrutia R, Kubes P: Platelets: bridging hemostasis, inflammation, and immunity. Int J Lab Hematol. 2013, 35:254-261. 10.1111/ijlh.12084

19. Gopi A, Madhavan SM, Sharma SK, Sahn SA: Diagnosis and treatment of tuberculous pleural effusion in 2006. Chest. 2007, 131:880-889. 10.1378/chest.06-2063

20. Heyderman RS, Makunike R, Muza T, et al.: Pleural tuberculosis in Harare, Zimbabwe: the relationship between human immunodeficiency virus, CD4 lymphocyte count, granuloma formation and disseminated disease. Trop Med Int Health. 1998, 3:14-20. 10.1046/j.1365-3156.1998.00167.x

21. Valdés L, Alvarez D, San José E, et al.: Tuberculous pleurisy: a study of 254 patients . Arch Intern Med. 1998, 158:2017-2021. 10.1001/archinte.158.18.2017

22. Lewinsohn DM, Leonard MK, LoBue PA, et al.: Official American Thoracic Society/Infectious Diseases Society of America/Centers for Disease Control and Prevention clinical practice guidelines: diagnosis of tuberculosis in adults and children. Clin Infect Dis. 2017, 64:111-115. 10.1093/cid/ciw778

23. Macías A, Sánchez-Montalvá A, Salvador F, Villar A, Tórtola T, Saborit N, Molina I: Epidemiology and diagnosis of pleural tuberculosis in a low incidence country with high rate of immigrant population: a retrospective study. Int J Infect Dis. 2019, 78:34-38. 10.1016/j.ijid.2018.10.005

24. Du J, Huang Z, Luo Q, et al.: Rapid diagnosis of pleural tuberculosis by Xpert MTB/RIF assay using pleural biopsy and pleural fluid specimens. J Res Med Sci. 2015, 20:26-31.

25. Luzze H, Elliott AM, Joloba ML, et al.: Evaluation of suspected tuberculous pleurisy: clinical and diagnostic findings in HIV-1-positive and HIV-negative adults in Uganda. Int J Tuberc Lung Dis. 2001, 5:746-753.

26. Casalini AG, Mori PA, Majori M, et al.: Pleural tuberculosis: medical thoracoscopy greatly increases the diagnostic accuracy. ERJ Open. Res. 2018, 4:00046-2017. 10.1183/23120541.00046-2017

27. Christopher DJ, Dinakaran S, Gupta R, James P, Isaac B, Thangakunam B: Thoracoscopic pleural biopsy improves yield of Xpert MTB/RIF for diagnosis of pleural tuberculosis. Respirology. 2018, 23:714-717. 10.1111/resp. 13275

28. von Groote-Bidlingmaier F, Koegelenberg CF, Bolliger CT, et al.: The yield of different pleural fluid volumes for Mycobacterium tuberculosis culture. Thorax. 2013, 68:290-292. 10.1136/thoraxjnl-2012-202338

29. Chaudhuri AD, Bhuniya S, Pandit S, Dey A, Mukherjee S, Bhanja P: Role of sputum examination for acid fast bacilli in tuberculous pleural effusion. Lung India. 2011, 28:21-24. 10.4103/0970-2113.76296

30. Conde MB, Loivos AC, Rezende VM, et al.: Yield of sputum induction in the diagnosis of pleural tuberculosis. Am J Respir Crit Care Med. 2003, 167:723-725. 10.1164/rccm.2111019 